\title{
Type-1.5 Superconductors
}

\author{
V. V. Moshchalkov, ${ }^{1}$ M. Menghini, ${ }^{1}$ T. Nishio, ${ }^{1}$ Q.H. Chen,${ }^{1}$ A. V. \\ Silhanek, ${ }^{1}$ V.H. Dao, ${ }^{1}$ L.F. Chibotaru, ${ }^{1}$ N. D. Zhigadlo, ${ }^{2}$ and J. Karpinski ${ }^{2}$ \\ ${ }^{1}$ INPAC-Institute for Nanoscale Physics and Chemistry, \\ Nanoscale Superconductivity and Magnetism \& Pulsed Fields Group, \\ K. U. Leuven Celestijnenlaan 200 D, B-3001 Leuven, Belgium. \\ ${ }^{2}$ Solid State Physics Laboratory ETH-Zürich, Switzerland.
}

(Dated: October 24, 2018)

\begin{abstract}
We demonstrate the existence of a novel superconducting state in high quality two-component $\mathrm{MgB}_{2}$ single crystalline superconductors where a unique combination of both type-1 $\left(\lambda_{1} / \xi_{1}<\right.$ $1 / \sqrt{2})$ and type-2 $\left(\lambda_{2} / \xi_{2}>1 / \sqrt{2}\right)$ superconductor conditions is realized for the two components of the order parameter. This condition leads to a vortex-vortex interaction attractive at long distances and repulsive at short distances, which stabilizes unconventional stripe- and gossamer-like vortex patterns that we have visualized in this type-1.5 superconductor using Bitter decoration and also reproduced in numerical simulations.
\end{abstract}


Vortices in superconductors are characterized by a normal core with size $\xi$ (coherence length) and supercurrents flowing over a distance $\lambda$ (penetration depth). If two vortices are generated in a type- 1 superconductor the normal cores would overlap first, due to the larger value of $\xi$ with respect to $\lambda$, thus leading to a gain in the condensation energy and, consequently, to vortex-vortex attraction[1, 2]. Two vortices in a type-2 material, however, would have first their supercurrents overlapping, in view of the bigger $\lambda$, leading to vortex-vortex repulsion. An attractive vortex-vortex interaction results in the formation of macroscopic normal domains in the intermediate state [3], while vortex-vortex repulsion leads to the appearance of the Abrikosov lattice. [4]

The recent discovery of the two-component superconductor $\mathrm{MgB}_{2}[5]$, with two weakly coupled coexisting order parameters, $\Psi_{1}=\Psi_{\pi}$ and $\Psi_{2}=\Psi_{\sigma}$, has opened remarkable new possibilities both for fundamental research and applications. Among the new research topics we find semi-Meissner state[6], the violation of the London law and Onsager-Feynman quantization[7], non-composite vortices [8], intrinsic Josephson effect with low-energy interband Josephson plasmons [9], mapping physics of two-component superconductors on two-condensate Bose systems [10], superfluidity in liquid metallic hydrogen [11], etc. The two-component character of $\mathrm{MgB}_{2}[12,13,14,15]$ is related with two different types of electronic bondings, $\pi$ and $\sigma$, giving rise to two different superconducting gaps with energies $\Delta_{\pi}(0)=2.2 \mathrm{meV}[16,17]$ and $\Delta_{\sigma}(0)=7.1 \mathrm{meV}$ [18, 19, 20], respectively. Using the BCS expression $\xi(0)=\hbar v_{F} / \pi \Delta(0)$, where $v_{F}$ is the Fermi velocity $\left(5.35 \times 10^{5} \mathrm{~m} / \mathrm{s}\right.$ for the $\pi$-band and $4.40 \times 10^{5} \mathrm{~m} / \mathrm{s}$ for the $\sigma$-band) [21] for the $\pi$ - and $\sigma$-bands separately, we obtain the two coherence lengths: $\xi_{\pi}(0)=51 \mathrm{~nm}$ and $\xi_{\sigma}(0)=13 \mathrm{~nm}$. The calculated $\xi_{\pi}(0)$ value is in agreement with $\xi_{\pi}(0)=49.6 \pm 0.9 \mathrm{~nm}$ obtained from the fit of the vortex profile measured by scanning tunneling spectroscopy (STS) [17]. The London penetration depths $\lambda_{\pi}$ and $\lambda_{\sigma}$ can be found from the respective plasma frequencies $\omega_{p \pi}$ and $\omega_{p \sigma}$ [22]: $\lambda_{\pi}(0)=33.6 \mathrm{~nm}$ and $\lambda_{\sigma}(0)=47.8 \mathrm{~nm}$. As a result, at least in the clean limit, the $\pi$ - and $\sigma$ - components of the order parameter in $\mathrm{MgB}_{2}$ are in different regimes: $\kappa_{\pi}=\lambda_{\pi}(0) / \xi_{\pi}(0)=0.66 \pm 0.02<1 / \sqrt{2}=0.707$ (type- 1$)\left(\right.$ assuming $\left.\lambda_{\pi}(0)=33.6 \pm 0.9 \mathrm{~nm}\right)$ and $\kappa_{\sigma}=\lambda_{\sigma}(0) / \xi_{\sigma}(0)=3.68>1 / \sqrt{2}$ (type-2). However, when $\mathrm{MgB}_{2}$ is doped with impurities, both components of the order parameter (due to an increase of $\lambda$ and a decrease of $\xi$ ) will fall inevitably into the type-2 regime. Therefore, clean $\mathrm{MgB}_{2}$ represents an excellent candidate to search for a new type of superconductivity, neither of type-1 nor type-2 
character, which we coined as type-1.5 superconductivity. In type-1.5 superconductors the vortex-vortex interaction is the result of the competition between short-range repulsion and long-range attraction and it is expected that vortices could form unusual patterns at low applied fields. [6]

In this Letter, we present experimental observation of vortex patterns at low vortex densities in high quality $\mathrm{MgB}_{2}$ single crystals. The vortex patterns are compared with the results of molecular dynamics simulations based on a two-gap Ginzburg-Landau (GL) theory which results in peculiar equilibrium vortex structures such as gossamer-like vortex patterns and vortex stripes.

The $\mathrm{MgB}_{2}$ single crystals were grown using a high pressure method as described elsewhere [23]. The critical temperature of the samples is typically $38.6 \mathrm{~K}$ as determined from the acsusceptibility response at zero field. The temperature dependence of the lower and upper critical fields $H_{c 1}(T)$ and $H_{c 2}(T)$ were determined by means of magnetization measurements [24, 25] and by ac-susceptibility measurements, respectively. From the extrapolated value $H_{c 2}(0)=5.1 \mathrm{~T}$ we obtain $\xi_{a b}(0)=8.0 \mathrm{~nm}$ close to the value found from BCS theory for $\xi_{\sigma}(0)$. In order to estimate the penetration depth of the $\sigma$-band we use the theoretical expression for the field at which the first vortex will penetrate a two component superconductor [Eq. (4) in Ref. [6]]. Considering $\lambda_{\pi}(0)=33.6 \mathrm{~nm}$ [22] for the type-1 component and taking into account $\xi_{a b}(0)=\xi_{\sigma}(0)=8.0 \mathrm{~nm}$ and $H_{c 1}(0)=0.241 \mathrm{~T}$ from our measurements we obtain $\lambda_{a b}=\lambda_{\sigma}=38.2 \mathrm{~nm}$ for our samples.

Coexisting two interpenetrating weakly coupled order parameters is a new quantum state with superflow of the two components through each other without any friction. A vortexvortex interaction is derived from the GL theory by numerically minimizing the free energy of two vortices with a variational procedure [26]. We use the two-band GL functional:

$$
F_{G L}\left[\Psi_{\sigma}, \Psi_{\pi}, \mathbf{A}\right]=F_{\sigma}+F_{\pi}+\int d^{3} r\left[\frac{E_{\gamma}}{2}\left(\Psi_{\sigma}^{*} \Psi_{\pi}+\Psi_{\pi}^{*} \Psi_{\sigma}\right)+\frac{1}{2 \mu_{0}}(\nabla \times \mathbf{A})^{2}\right]
$$

where

$$
F_{\alpha}=\int d^{3} r\left[2 E_{c \alpha}\left|\Psi_{\alpha}\right|^{2}+\left|E_{c \alpha}\right|\left|\Psi_{\alpha}\right|^{4}+\frac{\Phi_{0}}{2 \pi} \sqrt{\frac{\left|E_{c \alpha}\right|}{\mu_{0} \kappa_{\alpha}^{3}}}\left|\left(-i \nabla+\frac{2 \pi}{\Phi_{0}} \mathbf{A}\right) \Psi_{\alpha}\right|^{2}\right]
$$

where $\alpha=\sigma, \pi$ and $\Phi_{0}$ is the flux quantum. The estimations for the intrinsic condensation energies $E_{c \alpha}$ and the coupling energy $E_{\gamma}$ are taken from Ref. [27] and we use the values 
of $\kappa_{\sigma}$ and $\kappa_{\pi}$ obtained for our samples. The result of the minimization shows that the interaction between vortices is short-range repulsive and weakly long-range attractive, similarly to Ref. [6]. We model a system of overdamped vortices by molecular dynamics simulations. The equation of motion for a vortex $i$ is $\mathbf{F}_{i}=\mathbf{F}_{i}^{v v}+\mathbf{F}_{i}^{T}=\eta \mathbf{v}_{i}$, where $\mathbf{F}_{i}^{v v}$ represents the vortex-vortex interaction and $\mathbf{F}_{i}^{T}$ the thermal stochastic force satisfying $\left\langle\mathbf{F}_{i}^{T}(t)\right\rangle=0$ and $\left\langle\mathbf{F}_{i}^{T}(t) \mathbf{F}_{j}^{T}\left(t^{\prime}\right)\right\rangle=2 \eta \delta_{i, j} \delta\left(t-t^{\prime}\right) k_{B} T \cdot \eta\left(=\Phi_{0} H_{c 2} / \rho_{n}\right)$ is the viscosity, $H_{c 2}=5.1 \mathrm{~T}$ is the upper critical field and $\rho_{n}=0.7 \mu \Omega \cdot \mathrm{cm}$ [27] is the normal state resistivity. The system size used in our simulations is $2000 \times 2000 \lambda^{2}(0)$. Two systems with number of vortices $N_{v}=150$ and 400 are initially prepared in a high temperature molten state and then slowly annealed to $T=4.2 \mathrm{~K}$ with 1000 temperature steps. We let the system stabilize during 2000 time steps in each step of temperature. In order to compare the vortex structure of a type-1.5 with a conventional type-2 superconductor we also carry out this simulation for $\mathrm{NbSe}_{2}$ using the superconducting parameters from Ref. [28].

Bitter decoration experiments on $\mathrm{MgB}_{2}$ single crystals were performed at $4.2 \mathrm{~K}$ after cooling down the sample from above $T_{c}$ in the presence of an applied field perpendicular to the sample surface (field cooling, FC). In this way, a homogeneous vortex distribution all over the sample is expected. Details about the technique can be found elsewhere [29]. A Bitter decoration image at $H=1$ Oe shows clear evidence of an inhomogeneous distribution of vortices [Fig.1(a)] reminding gossamer patterns: local groups of vortices with intervortex distances shorter than the average vortex distance $\left(2 \Phi_{0} / \sqrt{3} B\right)^{1 / 2} \sim 5 \mu \mathrm{m}$ are separated by randomly distributed vortex voids with size of few micrometers. This is in striking contrast with the conventional homogenous vortex pattern formed in $\mathrm{NbSe}_{2}$ single crystals [see Fig.1(b)]. The observed clustering of vortices in $\mathrm{MgB}_{2}$ samples is consistent with the theoretical modeling made in Ref. [6] for a two-component superconductor in the semiMeissner state. In Fig.2(a) the vortex positions in a selected region of the Bitter decoration image shown in Fig.1(a) are indicated as white dots, while in Fig.2(b) the results from the numerical simulations for a two-component superconductor are shown. We also calculate the vortex configuration for a reference conventional type-2 superconductor. The obtained vortex structure, considering $\lambda=69 \mathrm{~nm}$ and $\xi=7.7 \mathrm{~nm}$ [28], is similar to the one observed in $\mathrm{NbSe}_{2}$ samples [see Figs.2(c) and (d)].

In order to characterize the inhomogeneity of the vortex structure (VS) we calculate the distribution of first neighbor distance, $P_{a}$ [Figs.2(e) and (f)]. The first neighbor distance, 
$a$, is calculated by means of the Delaunay triangulation of the vortex structure. For the VS of $\mathrm{NbSe}_{2}$, the distribution is Gaussian with a relative standard deviation $\delta=S D /<$ $a>=0.224$, where SD is the standard deviation of the Gaussian fit to the experimental data and $\langle a\rangle$ is the average first neighbor distance. On the other hand, in $\mathrm{MgB}_{2}$ samples $P_{a}$ is quite broad as a consequence of the inhomogeneous arrangement of vortices and has additional peaks at distances shorter and longer than the most probable separation [see the red and green arrows in Fig.2(e)]. The distribution of the first neighbor separation of the vortex structure obtained by simulations of a two-component material has also a three-peak structure which is fairly broader when compared to the one obtained in the case of a onecomponent conventional type-2 superconductor [Fig.2(f)]. The peaks at short distances in Figs.2(e) and (f) correspond to an average minimum separation between vortices.

Figure 3(a) shows a Bitter decoration image of the VS in $\mathrm{MgB}_{2}$ crystals at $H=5$ Oe and $T=4.2 \mathrm{~K}$. The vortex distribution appears also to be inhomogeneous at this field but in a rather different manner than in the experiment described above. In some regions of the sample, vortices agglomerate forming stripes while voids are formed on considerably larger areas. [30] The present experimental results seem to be in contradiction with previous reports of the vortex structure at low fields in $\mathrm{MgB}_{2}$ samples [31]. However, it is important to note that in Ref. 31 the authors show an image of the VS at 4 Oe in a very small region of the sample (approximately $10 \times 10 \mu \mathrm{m}^{2}$ ). Therefore, it is not possible to determine whether the vortex distribution in the samples studied in Ref. [31] is uniform all over the sample or not.

Although the vortex stripe pattern is rather disordered it is still possible to determine an average direction of vortex stripes as the one defined by the dashed yellow lines in Fig. 3(a). Considering this definition, we calculate the vortex density in lines parallel to the vortex stripes as a function of the distance measured along the direction of the yellow arrow in Fig.3(a). In the inset of Fig. 3(b) we plot the linear vortex density normalized by the average value for both the $\mathrm{MgB}_{2}$ and $\mathrm{NbSe}_{2} \mathrm{VS}$ at 5 Oe. In the case of $\mathrm{MgB}_{2}$, fluctuations of the vortex density of the order of $50 \%$ are observed. A similar calculation along lines perpendicular to the stripes shows that the standard deviation of the mean value is of the order of $30 \%$. The large fluctuations of the vortex density in $\mathrm{MgB}_{2}$ are in contrast to what is observed in $\mathrm{NbSe}_{2}$ crystals where the standard deviation of the vortex density is approximately $1 \%$ of the average value. A remarkable similarity is found between the experiments and simulations at still low density but higher than the one shown in 
Fig.2. Disordered vortex stripes are formed in the two-component superconductor while a homogeneous distribution is apparent in the case of a conventional type-2 material [Figs.3](c) and (d), respectively]. Consistently, the vortex density is seen to fluctuate in the direction perpendicular to the vortex stripes in the two-component material, as shown in the inset of Fig. [3(d).

Composition analysis (via an electron microprobe in a field emission scanning electron microscope) in an area across the stripes [in the direction of the white arrow in Fig. 3(a)] shows no significant variations in $\mathrm{Mg}$ or $\mathrm{B}$ content, thus ruling out the possibility to attribute the stripe formation to inhomogeneous surface pinning distribution. There is also no observed correlation between the vortex positions and localization of microdefects.

Bitter decoration images at $H=0.5$ Oe also show a very inhomogeneous vortex distribution (not shown). On the other hand, at $H=10$ Oe the VS in $\mathrm{MgB}_{2}$ samples is similar to the one in $\mathrm{NbSe}_{2}$ crystals indicating that this novel superconducting phase in two-component type-1.5 superconductors is only accessible at very low applied fields as predicted in Ref. [6].

In conclusion, the type-1.5 superconductivity is a totally new state which combines two regimes (type-1 and type-2) in the same single material (clean $\mathrm{MgB}_{2}$ and possibly also other two-gap materials such as $\mathrm{Ba}_{0.6} \mathrm{~K}_{0.4} \mathrm{Fe}_{2} \mathrm{As}_{2}$ [32]). The vortex matter in type-1.5 superconductors behaves in an extremely unusual way. The combination of the vortex-vortex repulsion and attraction in the same material leads to the appearance of novel vortex patterns: gossamer-like vortex arrays and vortex stripes. Both novel patterns have been directly visualized by Bitter decorations on high quality single crystals. Moreover, analytical modeling of exotic vortex-vortex interaction (attractive mixed with repulsive) and extensive molecular dynamic simulations are in good agreement with our experimental data.

We would like to thank P. L'Hoëst from the MTM Department in K. U. Leuven for performing the electron probe microanalysis experiments on the $\mathrm{MgB}_{2}$ crystals. This work was supported by Methusalem Funding of the Flemish government, FWO-Vlaanderen, and the Belgian Inter-University Attraction Poles IAP Programmes.

[1] L. Kramer, Phys. Rev. B 3, 3821 (1971).

[2] E. H. Brandt Phys. Rev. B 346514 (1986). 
[3] R. P. Huebener, Magnetic Flux Structures of Superconductors, (Springer-Verlag, New York, 1990).

[4] A. A. Abrikosov, Zh. Eksp. Teor. Fiz. 32, 1442 (1957).

[5] J. Nagamatsu et al., Nature 410, 63 (2001).

[6] E. Babaev and M. Speight, Phys. Rev. B 72, 180502(R) (2005).

[7] E. Babaev and N. W. Ashcroft, Nature Physics 3, 530 (2007).

[8] L. F. Chibotaru, V. H. Dao, and A. Ceulemans, Europhys. Lett. 78, 47001 (2007).

[9] G. Blumberg, et al., Phys. Rev. Lett. 99, 227002 (2007).

[10] E. Babaev, L. D. Faddeev, and A. J. Niemi, Phys. Rev. B 65, 100512(R) (2002).

[11] E. Babaev, A. Sudbø, and N. W. Ashcroft, Nature 431, 666 (2004).

[12] F. Bouquet, R. A. Fisher, N. E. Phillips, D. G. Hinks, and J. D. Jorgensen , Phys. Rev. Lett. 87, $047001(2001)$

[13] P. Szabo et al., Phys. Rev. Lett. 87, 137005 (2001).

[14] F. Giubileo et al., Phys. Rev. Lett. 87, 177008 (2001).

[15] S. Souma et al., Nature 423, 65 (2003).

[16] G. Rubio-Bollinger, H. Suderow, and S. Vieira, Phys. Rev. Lett. 86, 5582-5584 (2001).

[17] M. R. Eskildsen et al., Phys. Rev. Lett. 89, 187003 (2002).

[18] A. A. Golubov et al., J. Phys. Condens. Matter 14, 1353 (2002).

[19] H. J. Choi et al., Nature (London) 418, 758 (2002).

[20] M. Iavarone et al., Phys. Rev. Lett. 89, 187002 (2002).

[21] A. Brinkman et al. Phys. Rev. B 65, 180517(R) (2002).

[22] I. I. Mazin et al., Phys. Rev. Lett. 89, 107002 (2002).

[23] J. Karpinski et al., Supercond. Sci. Technol. 16, 221 (2003).

[24] V. V. Moshchalkov et al., Physica C 175, 407 (1991).

[25] G. K. Perkins et al., Supercond. Sci. Technol. 151156 (2002).

[26] L. Jacobs and C. Rebbi, Phys. Rev. B 19, 4486 (1979).

[27] M. Eisterer, Supercond. Sci. Technol. 20, R47 (2007).

[28] M. J. Higgins and S. Bhattacharya, Physica C 257, 232 (1996).

[29] Y. Fasano and M. Menghini, Supercond. Sci. and Tech. 21, 023001 (2008).

[30] It is important to note that this agglomeration in the form of vortex stripes is not related to the presence of steps or surface defects in the crystal. In fact, there are regions where steps 
can be observed (not shown) but at those locations the vortex configuration revealed by Bitter decoration is unaffected by them.

[31] L. Y. Vinnikov et al., Phys. Rev. B 67, 092512 (2003).

[32] H. Ding et al., Europhys. Lett. 83, 47001 (2008). 
FIG. 1: Magnetic decoration images of the vortex structure at $T=4.2 \mathrm{~K}$ and $H=1$ Oe. The vortices in the $\mathrm{MgB}_{2}$ single crystal (a) are distributed inhomogeneously while in the $\mathrm{NbSe}_{2}$ single crystal (b) they are arranged forming a disordered Abrikosov lattice. The scale bars in the images correspond to $10 \mu \mathrm{m}$. Notice that the density of vortices in the decoration experiments represents the internal field $B$ rather than the applied field $H$. This leads to different number of decorated vortices for $\mathrm{NbSe}_{2}$ and $\mathrm{MgB}_{2}$, even at the same applied field.

FIG. 2: (a) Experimental vortex locations in a selected part of the image shown in Fig. 1(a). The vortex configuration resulting from the numerical simulations in a two-component superconductor at low density is shown in (b) evidencing an inhomogeneous spatial distribution of vortices. In both cases, the regions enclosed by the dashed white line indicate voids of vortices caused by the inhomogeneous distribution. In (c) the vortex pattern obtained by a magnetic decoration of the $\mathrm{NbSe}_{2}$ crystal at 1 Oe is shown and (d) corresponds to the vortex pattern obtained by a numerical simulation of a type-2 superconductor. The white scale bars correspond to $10 \mu \mathrm{m}$. (e) and (f) display the distribution of first neighbor distance, $P_{a}$, of the experimental and theoretical vortex structures, respectively. The distributions obtained for the simulations of a type-2 material and for the $\mathrm{NbSe}_{2}$ vortex structure are Gaussian. In the case of $\mathrm{MgB}_{2} P_{a}$ shows additional peaks at distances shorter and longer than the most probable separation (see the red and green arrows). Pair of vortices separated at the distances where the additional peaks are located are highlighted in (a) and (b) by red and green circles. The light blue circles correspond to pair of vortices separated by the most probable separation. 
FIG. 3: (a) Magnetic decoration image showing a stripe-like vortex pattern in the $\mathrm{MgB}_{2}$ single crystal at $H=5$ Oe. (b)Disordered Abrikosov lattice with a homogeneous vortex density obtained at $H=5$ Oe in the $\mathrm{NbSe}_{2}$ sample. The formation of vortex stripes is also observed by numerical simulations of a two-component type-1.5 superconductor (c) in contrast to a homogeneous vortex distribution in a type- 2 superconductor at the same vortex density (d). The scale bars in the images correspond to $10 \mu \mathrm{m}$. Vortex density along lines parallel to the vortex stripe direction (yellow dashed lines in (a)) for $\mathrm{MgB}_{2}$ and $\mathrm{NbSe}_{2}$ vortex structures. The variation of the vortex density as a function of the distance measured along the direction perpendicular to the stripes (yellow arrows in (a) and (b)) is shown in the insert of (b). The curves are normalized by their respective average density. The results of a similar calculation performed on the simulated vortex structures are shown in the inset of $(d)$. 
This figure "Fig1.jpg" is available in "jpg" format from: http://arxiv.org/ps/0902.0997v3 
This figure "Fig2.jpg" is available in "jpg" format from: http://arxiv.org/ps/0902.0997v3 
This figure "Fig3.jpg" is available in "jpg" format from: http://arxiv.org/ps/0902.0997v3 